\title{
A novel method for severe plastic deformation at high strain rate
}

\author{
Harishchandra Lanjewar*, Leo Kestens, and Patricia Verleysen \\ EEMMeCS Department, MST-DyMaLab research group, Ghent University, Ghent, Belgium
}

\begin{abstract}
Severe plastic deformation (SPD) processing is defined as any method of forming under an extensive hydrostatic pressure that may be used to impart a very high strain to a bulk solid without any significant change in dimensions of the sample, producing exceptional grain refinement. Most of the SPD techniques employ very low processing speeds, however increased deformation rates are known to have a significant effect on the final microstructure. Most of the SPD processes operating at high rates do not impose hydrostatic pressures to the material and can therefore only be used for very ductile materials, while in others, the microstructural changes are limited to the surface layers of the material. To circumvent these restrictions a novel facility has been designed and developed where high hydrostatic pressures are maintained while a high shear deformation is imposed at high strain rates. The device combines the features of a high pressure torsion (HPT) unit with the principle of a torsional split Hopkinson bar (SHB) setup. A small ring-like sample, placed between two molds, is first subjected to a high, static pressure and subsequently to a high speed shear deformation upon release of torsional energy stored in a long bar. Although, the principle is rather straightforward, the design of the setup was extremely critical because of the high forces and energies involved. Tests have been performed on commercially pure aluminum. The material hardness increased in accordance with the microstructure and processing conditions; viz. annealed, only compressed and applied shear strain. Deformed grains departed from equiaxed shape and showed morphological texture in the direction of the shear even at very low strains indicating the presence of shear strains in the material. Further the material, or more specifically its mechanical properties and microstructure evolution is compared with conventional, statically deformed HPT samples.
\end{abstract}

\section{Introduction}

Grain refinement is known to introduce many remarkable properties in metals. SPD is one of the most successful ways to obtain fine-grained microstructures and is therefore researched extensively over last few decades. SPD treatments impart large plastic strains without much change in sample dimensions, thus paving the way for extreme grain fragmentation without failure in material. While many advances have been made in terms of number of methods imparting SPD, few studies exist where SPD is applied at high strain rates, although dynamic SPD is known to enhance structural fragmentation as well as to introduce new deformation mechanisms unavailable at slow strain rates [1]. Such a scheme of deformation is capable of reaching much finer structures compared to their slow rate deformation methods partially due to the suppression of thermally activated dislocation processes.

Most of the high strain rate severe deformation studies published so far rely on uniaxial compression facilities where deforming material is unconfined and is free to flow in other directions [2-7]. Such compression facilities are either of conventional upper-lower anvil type [2-4] or based on the split Hopkinson bar system [57]. Few of the studies involved application of torsional deformation using either a torsional Kolsky bar system [8-9] or conventional torsion deformation devices [10], again without any confinement of the deforming material which necessarily limits the amount of strain applied without material failure and can only be applied for materials with high intrinsic ductility. Ball milling [11], machining [12-13] and surface grinding methods [14] have also been explored in order to study the effects of a high strain rate deformation on the microstructure and properties of metals. However, in these methods microstructural changes are limited to the surface layers of the material and the cross-section of the deformed materials is insufficient for further mechanical property characterization. Additionally they only provide an opportunity to understand the evolution of the materials microstructure with strain rate, and are unusable as SPD metal forming operation in which a certain amount of material is deformed and fragmented into fine-structured material.

To overcome above mentioned limitations in existing methods, a novel facility for dynamic SPD has been designed and developed in present work. The facility has been used to deform commercially pure aluminium. The obtained microstructures and properties are presented, together with those of reference and statically deformed material.

Corresponding author: harishchandra.lanjewar@ugent.be 


\section{Experimental - Method and material}

Recently in the DyMaLab research group of Ghent University, a novel dynamic HPT (DHPT) setup has been developed and built. Its working principle is based on split Hopkinson bar torsion setups, which are used to characterise the mechanical behaviour of materials at high strain rates. Similar to conventional HPT, in the DHPT technique a disk-/ring-like sample is put between two molds, subjected to a static compression load and subsequently to a rotational deformation. Here the high strain rate shear deformation is imposed onto the material under semi-constrained conditions. A photo of setup is as shown in Fig. 1.

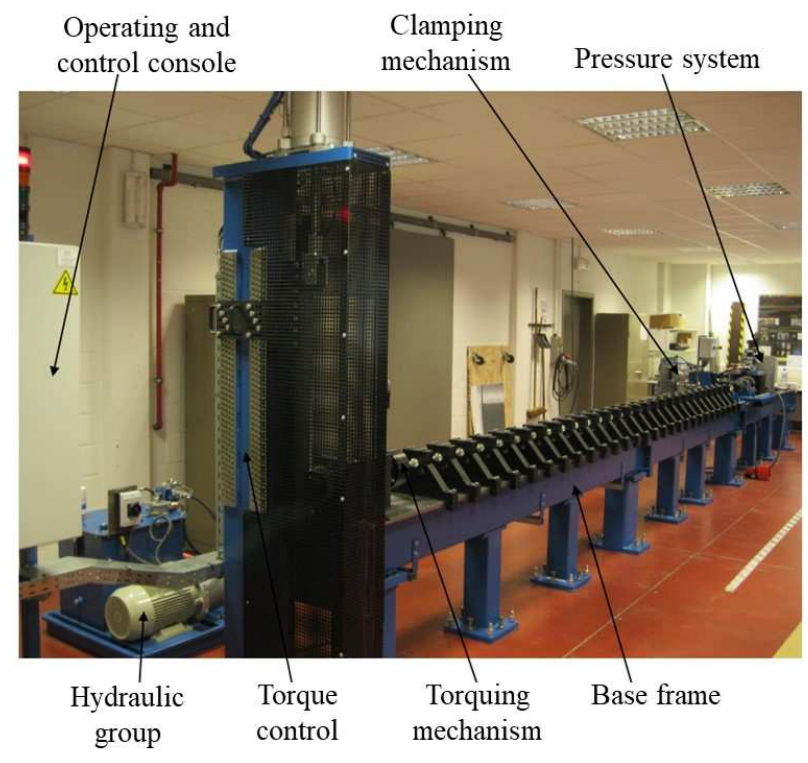

Fig. 1. A photo of the DHPT setup at Ghent University.

In this unit, a pneumatic system is used to apply the desired torque onto the axially compressed input bar while it is clamped at the other end using another pneumatic auxiliary unit. Once unclamped, the input bar releases a torsional plastic wave towards the specimen end, imparting shear deformation to the test material held under axial load, between two molds guaranteeing semi-constrained deformation conditions. The torsional wave in the input bar gives rise to a rotational speed of one of the molds, which in turn determines the strain rate applied to the material sample in between the molds. Indeed as is the case for conventional HPT, the sample geometry and the number of applied turns can be used to calculate the strain imposed on the sample using the equation for equivalent von Mises strain, $\varepsilon_{e q}$ :

$$
\varepsilon_{e q}=\gamma / \sqrt{ } 3=\left(2 \pi N \cdot r_{s}\right) /\left(\sqrt{ } 3 \cdot L_{s}\right)
$$

where $\gamma$ is the engineering shear strain imposed on a sample with radius $r_{s}$ and height $L_{s}$, and $N$ the number of applied revolutions.

Commercial purity aluminium was selected as sample material to conduct initial experiments, since numerous results were available of static HPT tests on this material [15-17]. The as received material had the following composition: $\mathrm{Al}-0.28 \% \mathrm{Fe}-0.05 \% \mathrm{Si}-0.05 \% \mathrm{Cu}$ (wt $\%$ ), and was cold rolled and annealed. The ringshaped specimens were produced having an internal diameter of $5 \mathrm{~mm}$, an outer diameter of $11 \mathrm{~mm}$ and 1 $\mathrm{mm}$ thickness.

In order to compare the performance of DHPT processed material, with that of conventional, static HPT, disk shaped specimen with $15 \mathrm{~mm}$ diameter were processed using HPT at small rotations of 10, 15, 20, and 25 degrees. The deformed samples were investigated for their microstructure and mechanical properties using micro-hardness testing, optical microscopy and orientation imaging microscopy (OIM) using EBSD. TSL OIM software was used for microstructural and texture analysis.

The geometry and reference system common for both disk- and ring-specimens are shown in Fig. 2, together with the surface used for optical and OIM investigations. For micro-hardness measurements the $r-\theta$ plane was considered.

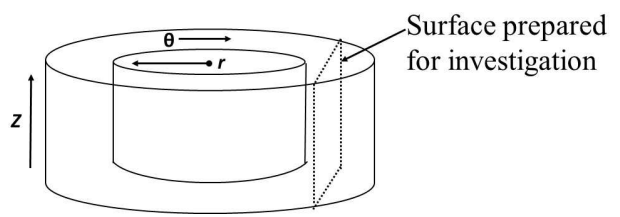

Fig. 2. Geometry of the ring-specimen with $\theta$ being the shear direction (SD), $r$ the radial direction (RD) and $Z$ the shear plane normal (SPN). The indicated $\theta-Z$ plane was prepared for optical and OIM investigation.

\section{Result and discussion}

The initial material showed completely recrystallized and equiaxed microstructure as shown with the EBSD colour map in Fig. 3 displaying grain boundaries (GBs) between $15^{\circ}-65^{\circ}$ in black colour. The recrystallized structure had a mean grain size of $\sim 85 \mu \mathrm{m}$, with almost

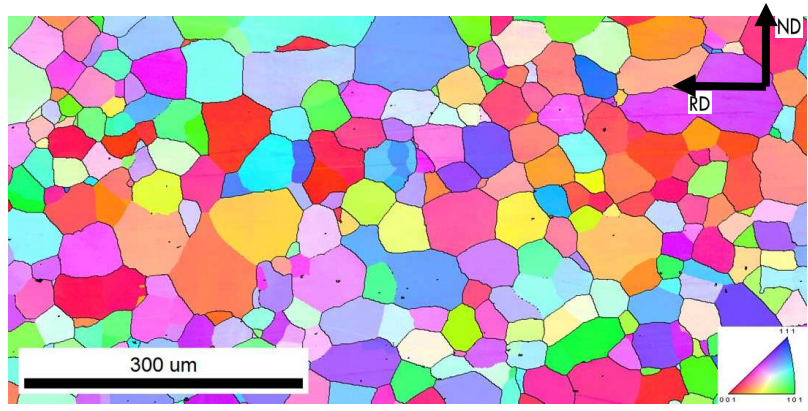

Fig. 3. EBSD colour map of the annealed aluminium.

$87 \%$ of the total grain boundary lengths as high angle GBs (HAGBs). Low angle grain boundaries between $5^{\circ}$ $15^{\circ}$ accounted for the remaining $13 \%$ while no boundaries below $5^{\circ}$ misorientation angle were observed. The microtexture was dominated by the cube and $\mathrm{R}$ orientations accompanied with retained rolling components $\mathrm{Cu}\{112\}<111>$ and $\mathrm{S}\{231\}<346>$ (shown in Fig. 7) [18].

Fig. 4 shows the optical microscopy image of a DHPT deformed sample in a $\theta-Z$ plane, with the area 
enclosed by a white dotted circle also shown in a magnified image. On application of axial load and subsequent shear loading of the sample, material flows out between the two mold surfaces, and thus provides the hydrostatic condition necessary for continuing shear deformation of material in the mold cavities. The outward material flow can be observed from the elongated grains at the centre plane across the thickness of the sample in magnified image. The area encircled with a black dotted circle in the magnified image shows equiaxed grains almost undeformed or having undergone very little deformation owing to the dead zone and thus affecting the homogeneity of hardness across the thickness of the sample [19].

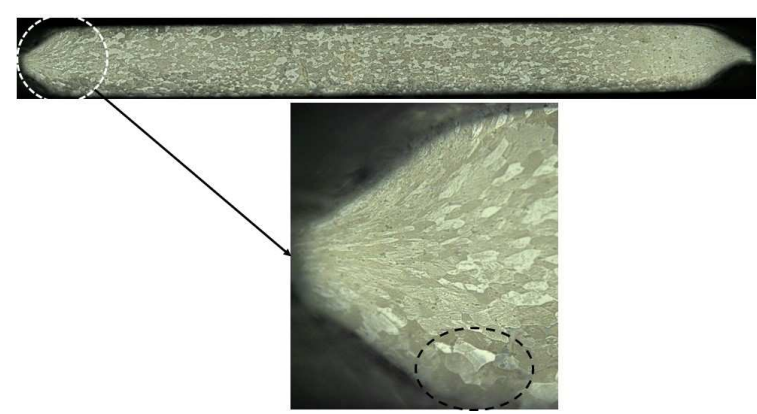

Fig. 4. Optical microscopy image of a sample deformed via DHPT to a rotation of $5 \mathrm{deg}$, with the corner of the sample (within the white circle) magnified in the image below it.

The OIM IPF maps for HPT and DHPT deformed samples are shown in Fig. 5. In order to perform statistically reliable analysis of various microstructure and texture parameters, OIM scan data was collected from at least 500 grains for each condition. OIM data cleanup involved elimination of data points with confidence index lower than 0.1 , followed by single step grain dilation correction. The minimum grain tolerance angle of $15^{\circ}$ was chosen for defining a grain, consisting of at least 5 pixels.

From above IPF maps a certain directionality in the flow of grains can be discerned. This morphological texture in grains coincides with the direction of applied shear. In case of static HPT, the deformation was imposed anti-clockwise, i.e. the moving part of the mold rotated in anti-clockwise direction; while during DHPT processing the shear was applied in clockwise direction, with reference to the fixed plane of the sample.

Additionally, a gradient in the colours of the grains can be observed in the deformed samples. In annealed condition, grains are coloured more uniformly. This indicates the introduction of substructure within grains and thereby spread in orientation. The samples presented in Fig. 5 (a) and (b), although strained to similar strains, the DHPT sample displayed slightly more orientation spread within grains. With increase in equivalent strain during DHPT, increased fragmentation in the structure can be observed.

The mean grain size analysis was performed using the OIM data and is plotted in Fig. 6 below. For the mean grain size analysis, a minimum boundary misorientation cut-off of $2^{\circ}$ was considered and $15^{\circ}$ for HAGBs. As can be seen, in the static HPT sample, the
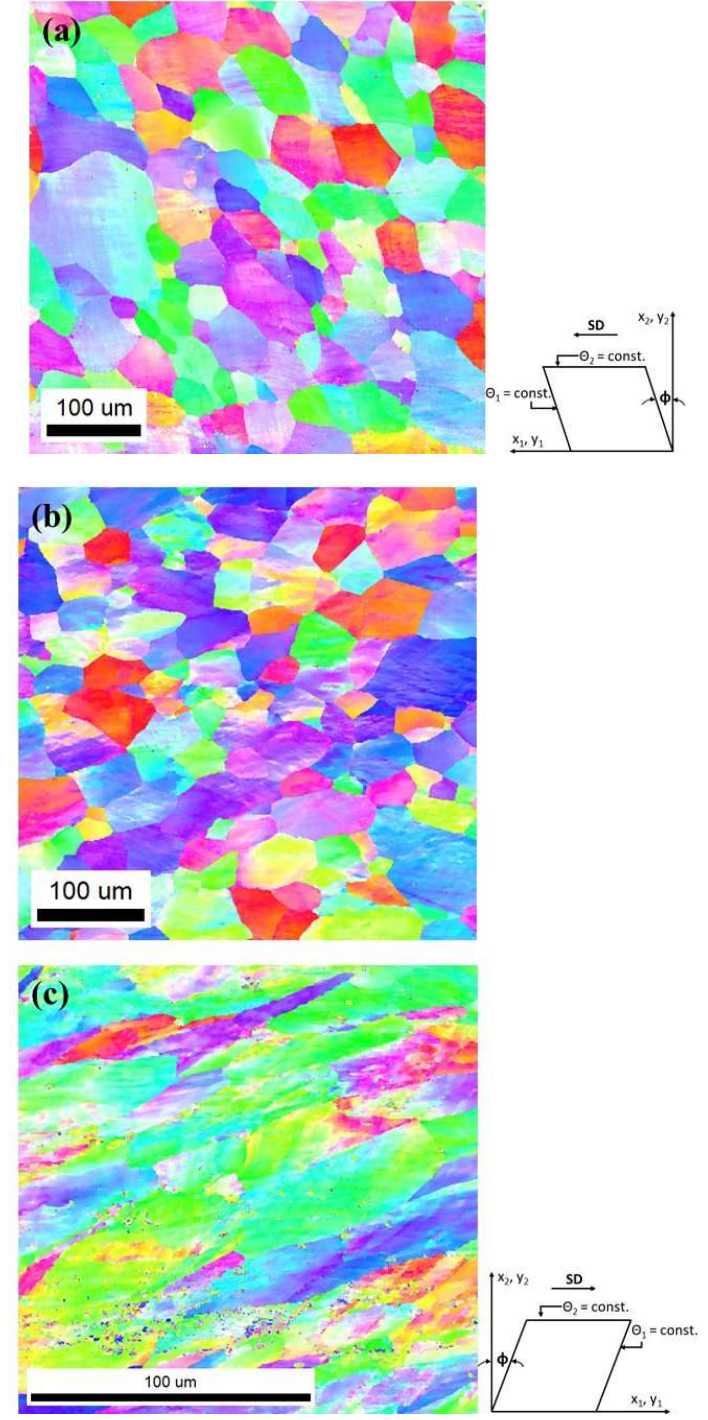

Fig. 5. OIM inverse pole figure (IPF) map for (a) HPT deformed -0.21 , (b) DHPT deformed -0.21 , and (c) DHPT deformed -1.34 equivalent strain; along with the respective SDs denoted in the deformation mode key beside it.

mean grain size hardly changed from that in annealed condition, whereas in similarly deformed DHPT sample, the grain size reduced by $\sim 10 \%$, demonstrating initiation of the grain fragmentation process which continues with increase in shear strain.

In texture analysis the grain orientation is always expressed in terms of an external co-ordinate system. In case of cold rolled and recrystallized material, this external reference frame conventionally consists of the rolling direction (RD), the normal direction (ND) and the transverse direction (TD). Then any crystal orientation described using miller indices (hkl)[uvw] means that a plane (hkl) is parallel with the rolling plane and the direction [uvw] is parallel with the RD. On the other hand, in case of torsion texture notations (hkl)[uvw] means that (hkl) is parallel to the shear plane $r-\theta$, and $[\mathrm{uvw}]$ is parallel to the SD. 


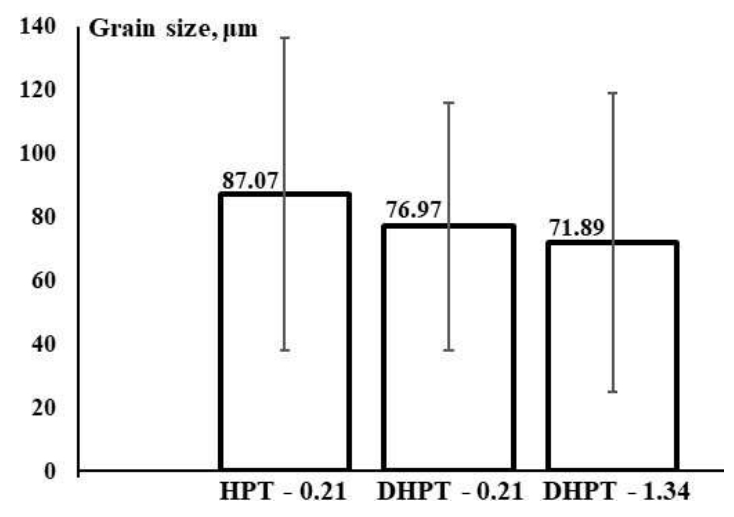

Fig. 6. Grain size obtained in different processing conditions.

The orientation data obtained from EBSD was used to calculate the pole figures and subsequently the texture index. A harmonic series method with series rank (L) of 16 , and Gaussian half-width of 7.5 was used. In case of annealed material orthotropic symmetry was imposed while for shear texture calculation triclinic symmetry was used.
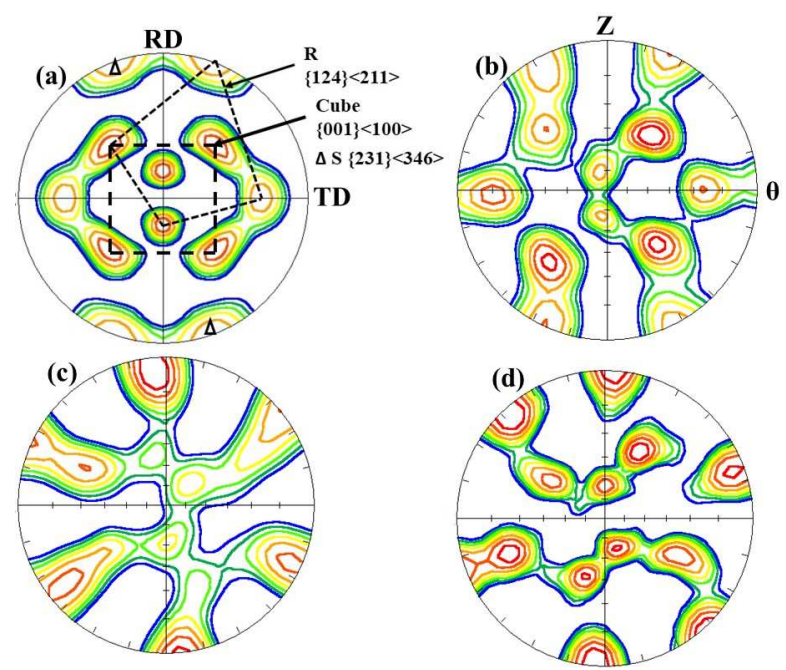

Fig. 7. (111) pole figures calculated for (a) annealed, (b) DHPT -0.21 , (c) DHPT -1.34 , and (d) HPT -0.21 equivalent strain samples. Ideal orientations for recrystallized commercial purity aluminium are also marked in (a).

Fig. 7 shows (111) pole figures calculated for annealed, and SPD deformed samples. As described earlier, the pole figure for annealed materials shows presence of recrystallization and retained rolling components. At small strains of SPD, the annealed texture continues to exist in both DHPT (Fig. 7 (b)) and static HPT (Fig. 7 (d)) deformed specimen, with recrystallization orientations slightly moving away from their ideal positions. However, with increase in imposed DHPT strain, ideal orientations of torsion texture start to appear in Fig. 7 (c) [20].

In order to understand the effect of SPD processing on the evolution of texture strength, the texture index (TI) was calculated. It represents the integral of the square of the orientation distribution function. A random textured material has a TI of unity, while a single component would have an infinite value [21]. Fig. 8 shows the TI values calculated for all the SPD processed conditions. The recrystallized material possessed a TI value of 4.91 [16], which decreases to a value of 2.2 for the static HPT sample and 1.94 for the dynamic HPT sample. The dynamic HPT sample with $\varepsilon_{e q}$ of 1.34 shows a TI value of 1.74. The weakening of texture is the direct result of the observed grain fragmentation. The newly formed grains develop from existing grains but do not necessarily have the same orientation and hence a decreasing TI is witnessed.

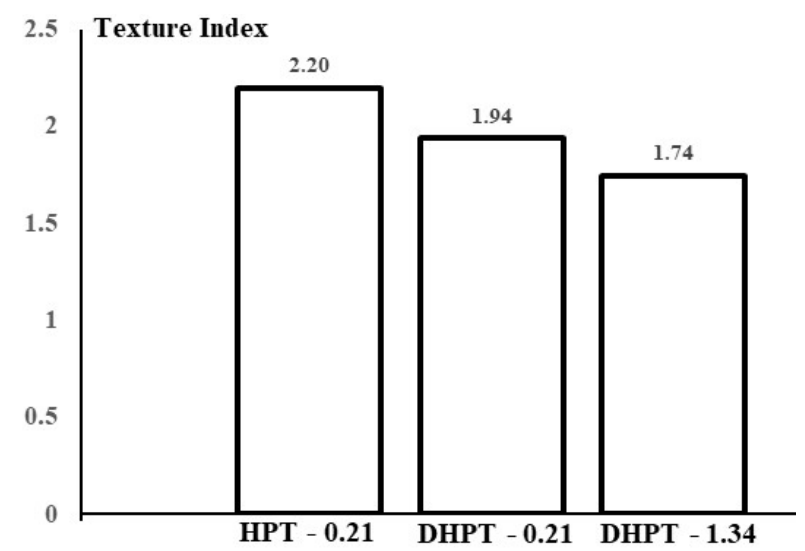

Fig. 8. Texture index calculated for SPD processed materials.

In order to correlate the modifications observed in the microstructure, micro-hardness measurements were performed. The indentation load of $100 \mathrm{gf}$ was applied for $15 \mathrm{~s}$ and an average of three indentations was reported as the mean hardness value. In order to inspect the effect of orientation over hardness, in DHPT sample measurements were performed along $0, \pm 45$, and $90 \mathrm{deg}$. In the static HPT sample, measurements were performed along 0 and $90 \mathrm{deg}$. The annealed and only compressed (at $1.33 \mathrm{GPa}$ ) materials exhibited hardness values of 24 and $35 \mathrm{HV}_{0.1}$, respectively. In DHPT sample hardness values along different orientations almost overlapped each other and reached a maximum value of $55 \mathrm{HV}_{0.1}$ at an imposed strain corresponding with $\varepsilon_{e q}$ of 0.91 , as shown in Fig. 9 (a). Fig. 9 (b) shows the hardness evolution in HPT samples, statically deformed to rotations of $10,15,20$ and $25 \mathrm{deg}$. The hardness measurements follow a straight line pattern with increase in equivalent strain. It should be noted that the static HPT samples are able to reach a value of $55 \mathrm{HV}_{0.1}$ only at an $\varepsilon_{e q}$ of 1.14. Thus it can be concluded that the hardness evolution in static HPT samples is slightly slower than in dynamic HPT samples. 

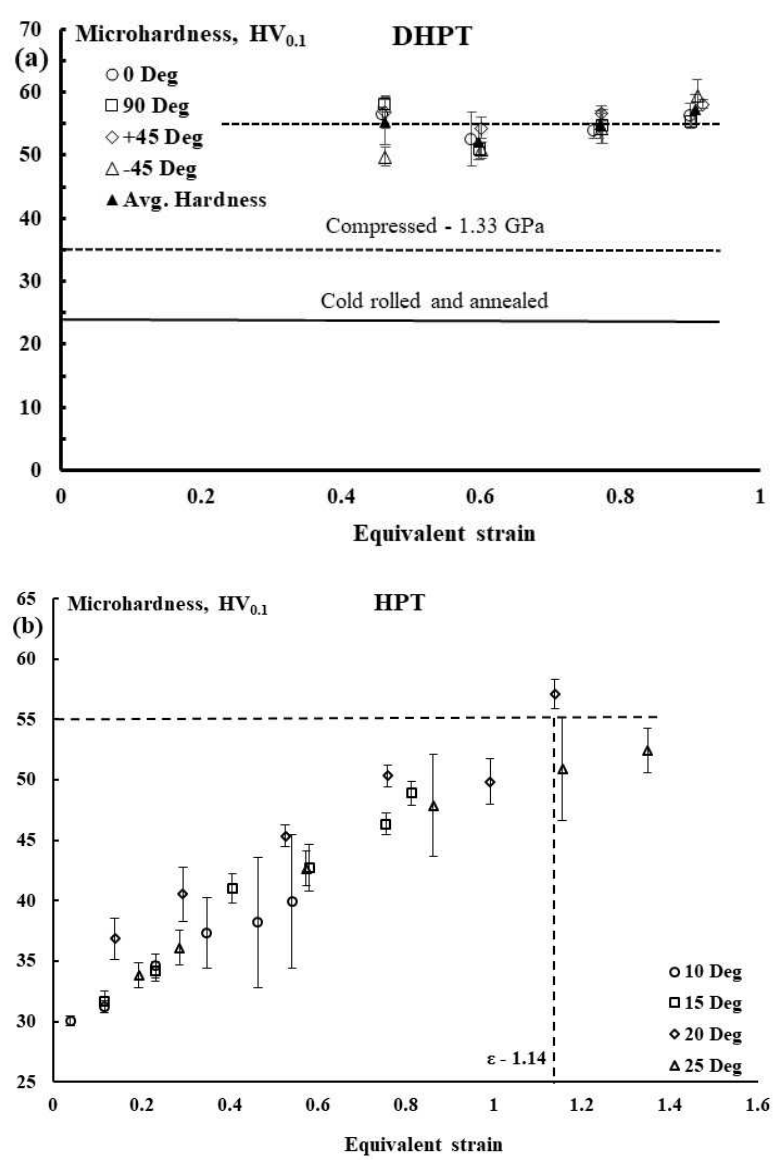

Fig. 9. Micro-hardness measurements for (a) DHPT sample and (b) HPT samples is plotted vs. equivalent SPD strain.

\section{Summary}

In present work, a dynamic SPD facility is introduced which combined the principles of split Hopkinson torsion bar systems and HPT devices in order to impart shear deformation onto the material at high strain rates. Commercially pure aluminium samples were deformed under both static and dynamic HPT conditions, and it was observed that grain fragmentation was slightly faster in dynamic HPT samples than in static HPT samples. Correspondingly, the hardness evolution was also found to be slower in static HPT samples. Though in the results presented above, the differences between the static and dynamically deformed samples are marginal, it should be noted that the samples were deformed to very low strain values. At higher strains this gap may widen, partially owing to the introduction of new deformation mechanisms.

In present investigation, the dynamic HPT strains were limited due to considerable slippage of sample within molds at high rotation speeds. Further work involves taking countermeasures to reduce the slippage. However, it should be kept in mind that large deformations at high strain rate means significant adiabatic heating which will promote the recovery of deformation structures. Therefore, further DHPT tests will be performed with a stepwise introduction of the strain. In between the deformation steps, the sample will be allowed to cool down.

\section{References}

1. G. T. G. III, Annu. Rev. Mater. Res. 42, 285-303 (2012)

2. Y. S. Li, Y. Zhang, N. R. Tao and K. Lu, Acta Mater. 57, 761-772 (2009)

3. G. Dirras, T. Chauveau, A. Abdul-Latif, J. Gubicza, S. Ramtani, Q. Bui, Z. Hegedus and B. Bacroix, Metall. Mater. Trans. A 43, 1312-1322 (2012)

4. B. Zhang and V. P. W. Shim, Acta Mater. 58, 68106827 (2010)

5. D.-H. Ahn, D. J. Lee, M. Kang, L. J. Park, S. Lee and H. S. Kim, Metall. Mater. Trans. A 47, 16001606 (2016)

6. B. K. Kad, J.-M. Gebert, M. T. Perez-Prado, M. E. Kassner and M. A. Meyers, Acta Mater. 54, 41114127 (2006)

7. A. Tiamiyu, R. Basu, A. G. Odeshi and J. A. Szpunar, Mater. Sci. Eng. 636, 379-388 (2015)

8. S. Khamsuk, N. Park, S. Gao, D. Terada, H. Adachi and N. Tsuji, Mater. Trans. 55, 106-113 (2014)

9. S. Khamsuk, N. Park, H. Adachi, D. Terada and N. Tsuji, J. Mater. Sci. 47, 7841-7847 (2012)

10. C. Y. Chiem and J. Duffy, Mater. Sci. Eng. 57, 233247 (1983)

11. H. Inomoto, H. Fujiwara and K. Ameyama, in NANOSPD2, Vienna, (2002)

12. T. L. Brown, C. Saldana, T. G. Murthy, J. B. Mann, Y. Guo, L. F. Allard, A. H. King, W. Dale Compton, K. P. Trumble and S. Chandrasekar, Acta Mater. 57, 5491-5500 (2009)

13. S. Shekhar, J. Cai, S. Basu, S. Abolghasem and M. Ravi Shankar, J. Mater. Res. 26, 395-406 (2011)

14. P. Xu, H. Luo, Z. Han and J. Zou, Mater. Des. 85, 240-247 (2015)

15. S. Naghdy, L. L. Percq, R. Serret, R. Petrov, S. Hertelé, L. Kestens and P. Verleysen, Mater. Sci. Tech. 33, 678-687 (2016)

16. S. Naghdy, L. Kestens, S. Hertelé and P. Verleysen, Mater. Charact. 120, 285-294 (2016)

17. S. Naghdy, H. Pirgazi, P. Verleysen, R. Petrov and L. Kestens, J. Appl. Crystallogr. 50, 1512-1523 (2017)

18. Samajdar, B. Verlinden, L. Rabet and P. Van Houtte, Mater. Sci. Eng. A 266, 146-154 (1999)

19. Hohenwarter, A. Bachmaier, B. Gludovatz, S. Scheriau and R. Pippan, Int. J. Mater. Res. 100, 1653-1661 (2009)

20. L. S. Tóth, J. J. Jonas, D. Daniel and J. A. Bailey, Textures Microstruct. 19, 245-262 (1992)

21. U. F. Kocks, C. N. Tome and H. -R. Wenk, Texture and anisotropy: Preferred orientations in polycrystals and their effect on materials properties, Cambridge: Cambridge university press (2000) 
